\title{
APEX and ATCA observations of the southern hot core G327.3-0.6 and its environs
}

\author{
Friedrich Wyrowski • Per Bergman • Karl Menten • \\ Jürgen Ott · Peter Schilke • Sven Thorwirth
}

Received: 31 January 2007 / Accepted: 9 July 2007 / Published online: 3 August 2007

(c) Springer Science+Business Media B.V. 2007

\begin{abstract}
There is no generally accepted evolutionary scheme for high mass star formation yet. A simple approach to address this problem is to cover several of the known stages during the formation of massive stars in the same cloud and then investigate their properties trying to construct an evolutionary sequence. Here we present such a project conducted with complementary APEX and ATCA observations. These observations show a compact and bright single hot core in the G327.3-0.6 region on a 0.03 pc scale with a mass of $500 \mathrm{M}_{\odot}$ and $0.5-1.510^{5} \mathrm{~L}_{\odot}$. Additionally a clumpy filament is seen in $\mathrm{N}_{2} \mathrm{H}^{+}$. Together with cm continuum observations, the data reveal like pearls on a string several stages of massive star formation, with likely the youngest stages hiding in the cold $\mathrm{N}_{2} \mathrm{H}^{+}$cores analysed with a multilevel study of the APEX and ATCA observations.
\end{abstract}

Keywords G327.3-0.6 · Massive star formation

F. Wyrowski $(\bowtie) \cdot$ K. Menten $\cdot$ P. Schilke $\cdot$ S. Thorwirth Max-Planck-Institut für Radioastronomie, Bonn, Germany e-mail: wyrowski@mpifr-bonn.mpg.de

P. Bergman

European Southern Observatory, Santiago, Chile

P. Bergman

Onsala Space Observatory, 43992 Onsala, Sweden

J. Ott

National Radio Astronomy Observatory, Charlottesville, VA, USA

\section{High mass star formation: the quest for an evolutionary scheme}

The quest for an evolutionary scheme, comparable to the CLASSes framework that exists for the early evolution of low-mass protostars, is currently one of the main topics of research in the field of high mass star formation. In analogy to low mass prestellar cores, precluster cores are expected in the high mass case which are cold $(\leq 20 \mathrm{~K})$ and massive $\left(\sim 100-1000 \mathrm{M}_{\odot}\right)$. The observational evidence for these cores is still scarce. Massive young stellar objects will eventually start to build up mass within these cores and develope from a still relatively cold and infrared dark phase into hot molecular cores. These are internally heated to temperatures high enough to evaporate grain mantles $(T>100 \mathrm{~K})$ and are dense $\left(n \sim 10^{7} \mathrm{~cm}^{-3}\right.$ ) with infall still ongoing, so that HII regions can be quenched for some time (Walmsley 1995) before observable hyper- and ultra-compact HII region form around the young massive cluster.

This evolutionary scheme can be studied by either surveys of a large sample of young massive star forming regions selected to cover a wide range in evolutionary phases (see for example Hieret et al. this volume) or by studying in detail template regions, which harbor several of these phases simultaneously. An example of such a region is the giant molecular cloud associated with the bright southern hot core G327.3-0.6, which has the potential of becoming a southern hemisphere hot core template for upcoming observatories like ALMA and was therefore studied by us with APEX (Wyrowski et al. 2006) and ATCA (Wyrowski et al., in preparation). 


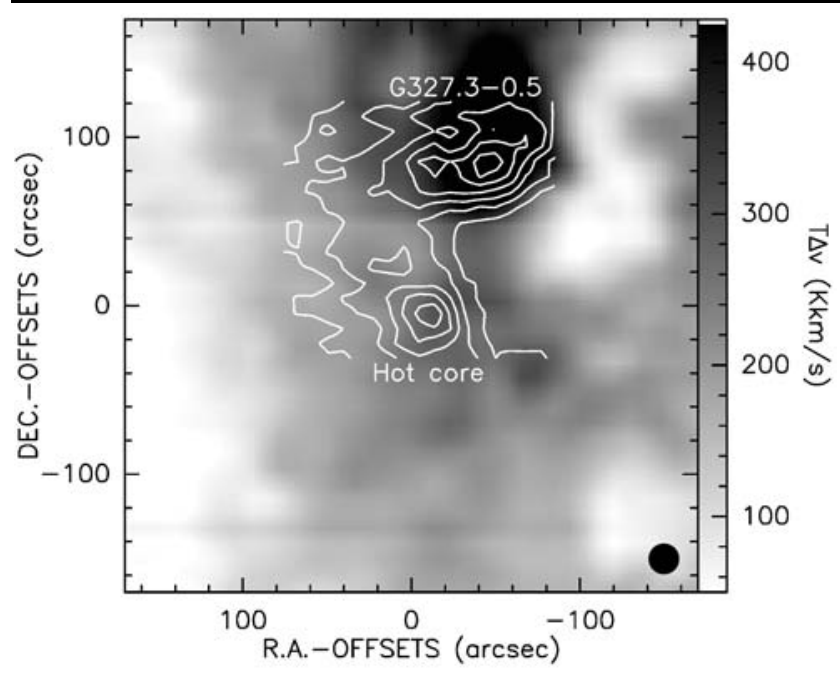

Fig. 1 APEX ${ }^{12} \mathrm{CO}$ (greyscale) and $C^{18} \mathrm{O}$ (3-2) (contours) images. The contour levels start at 23.6 with steps of $15.6 \mathrm{~K} \mathrm{~km} / \mathrm{s}$

\section{The massive star forming region G327.3-0.6}

The hot molecular core associated with the HII region G327.3-0.6 is situated at a kinematical distance of $2.9 \mathrm{kpc}$ (Bergman 1992) about 2 arcmin south of a bright complex of infrared sources. It was discovered by its association with prominent $\mathrm{H}_{2} \mathrm{O}, \mathrm{OH}$, and $\mathrm{CH}_{3} \mathrm{OH}$ masers, and its chemistry has been studied in two papers, one reporting ethylene oxide and acetaldehyde observations (Nummelin et al. 1998), while the other investigates the chemical inventory of this source (Gibb et al. 2000). The source is remarkable for its exceptionally rich molecular line spectra with relatively narrow, well-behaved (Gaussian) line profiles (Schilke et al. 2006). Compared to hot molecular cores accessible from the northern hemisphere almost nothing is known about its environs. Only Bergman (1992) reports some SEST maps that reveal two adjacent dense cores in this molecular cloud: one relatively cold ( $\left.T_{\text {kin }} \sim 30 \mathrm{~K}\right)$ molecular clump and one hot ( $T=100-200 \mathrm{~K})$ core. Hence, this region offers the possibility to study cores that have formed from the same parental cloud, but that are in different stages of evolution.

\section{Observations}

Single dish observations of G327.3-0.6 were done with the Atacama Pathfinder Experiment (APEX, Güsten et al. 2006). To probe the large scale environs of the sources, arcmin ${ }^{2}$-sized On-The-Fly maps were observed in ${ }^{12} \mathrm{CO}$ and $\mathrm{C}^{18} \mathrm{O}$ (3-2) (see Fig. 1). A smaller part of the cloud was observed with raster mapping in $\mathrm{N}_{2} \mathrm{H}^{+}(3-2)$. Higher $\mathrm{N}_{2} \mathrm{H}^{+}$ transitions were observed towards the peak of the $\mathrm{N}_{2} \mathrm{H}^{+}$ (3-2) emission.

Interferometric observations of the source at $3 \mathrm{~mm}$ were performed with the Australia Telescope Compact Array

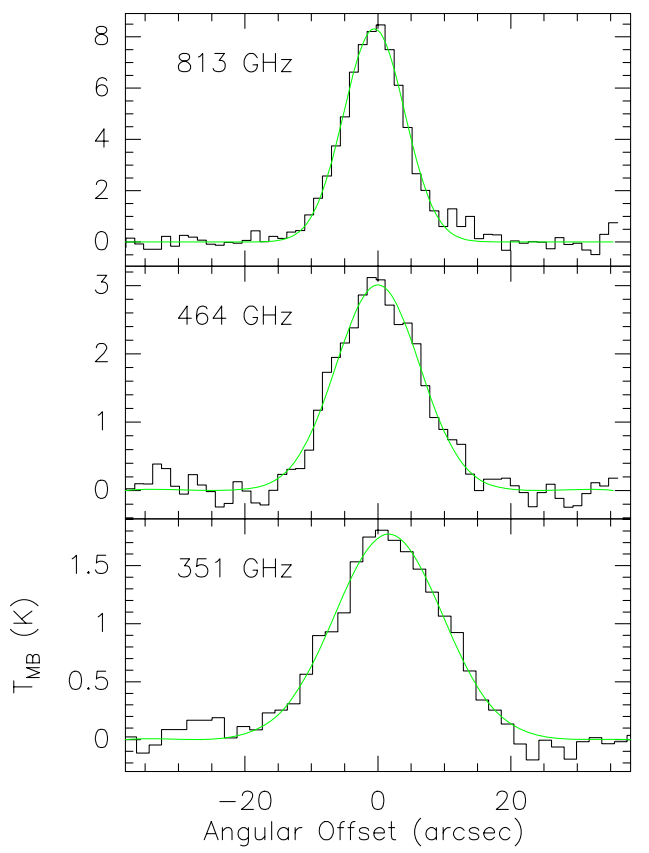

Fig. 2 APEX Continuum scans of the hot molecular core

(ATCA) in the $\mathrm{H} 75$ and $\mathrm{H} 214$ configurations, resulting in an angular resolution down to $2^{\prime \prime}$. A 4 point mosaic was observed to cover the cold and the hot core. $\mathrm{N}_{2} \mathrm{H}^{+}(1-0)$ was observed with a spectral resolution of $0.2 \mathrm{~km} / \mathrm{s}$ and the $3 \mathrm{~mm}$ continuum and the (5-4) $\mathrm{K}$ ladder of $\mathrm{CH}_{3} \mathrm{CN}$ were observed with 32 channels and a total bandwidth of $128 \mathrm{MHz}$.

\section{The hot molecular core}

The large scale CO emission as observed with APEX is shown in Fig. 1. The hot molecular core, while relatively inconspicuous in ${ }^{12} \mathrm{CO}$, shows up as a strong column density peak in $\mathrm{C}^{18} \mathrm{O}$. In the northern part of the map strong $\mathrm{CO}$ emission traces the hot surface of a bright photon dominated region associated with the strong HII region G327.30.5 and the complex of infrared sources around IRS3 (Goss and Shaver 1970; Epchtein and Lepine 1981). The mass of the core derived from $\mathrm{C}^{18} \mathrm{O}$ is about $500 \mathrm{M}_{\odot}$.

Simultaneously with $\mathrm{C}^{18} \mathrm{O}, \mathrm{CH}_{3} \mathrm{OH} 7_{1}-6_{1} A^{-}$was observed, which is expected to originate from hot molecular gas. Its peak is aligned with the $\mathrm{C}^{18} \mathrm{O}$ column density peak. Towards this position, a typical line-rich hot core spectrum is observed (Fig. 3, lower panel). Continuum cross scans with APEX (Fig. 2) peak also at the $\mathrm{CH}_{3} \mathrm{OH}$ and $\mathrm{C}^{18} \mathrm{O}$ peaks and can be used to derive a dust mass of the core. Depending on the assumed dust properties, the dust mass range from 400 to $1000 \mathrm{M}_{\odot}$, consistent with the $\mathrm{C}^{18} \mathrm{O}$ derived value. The $\mathrm{mm} / \mathrm{submm}$ continuum fluxes can be used together with infrared flux densities to construct the spectral energy distribution of the sources. With a 20 and 100 micron fluxes from 


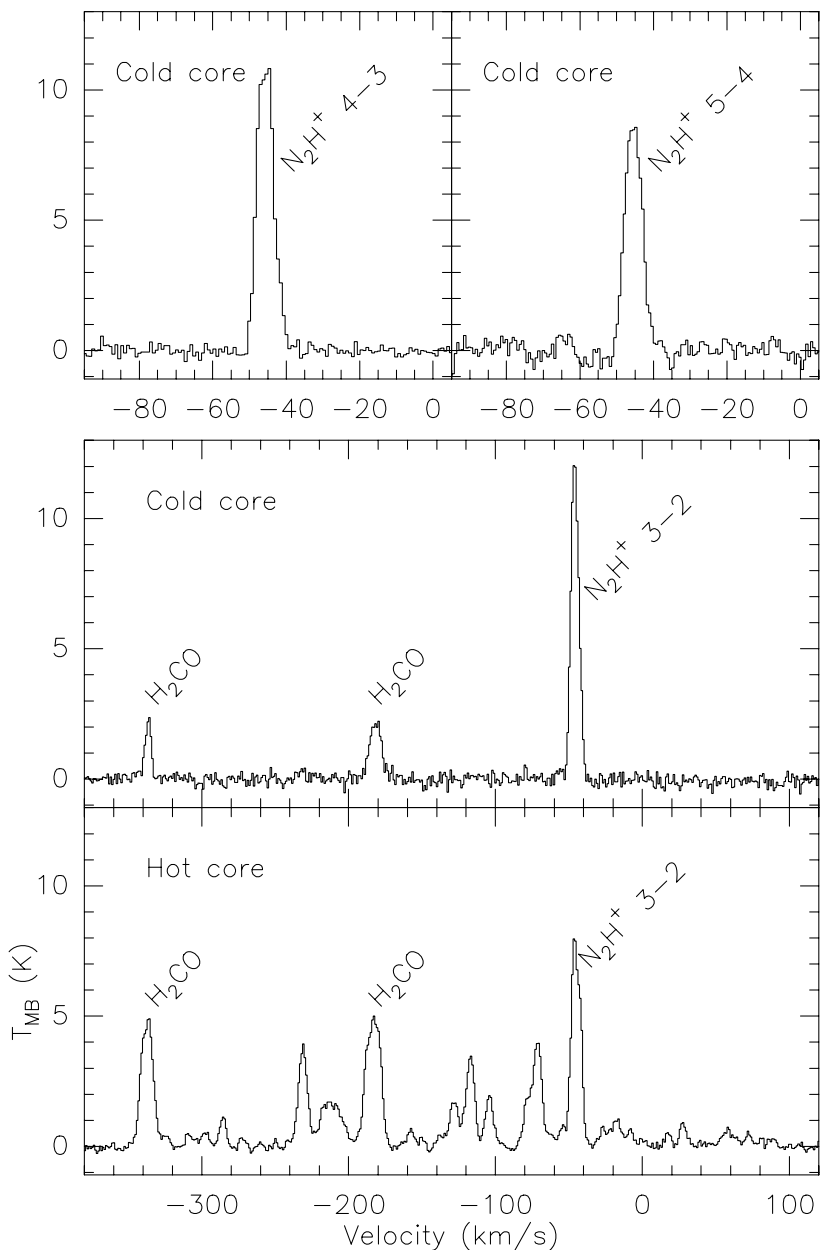

Fig. 3 APEX spectra observed toward the cold core $\left(\left(12^{\prime \prime}, 6^{\prime \prime}\right)\right.$, upper panels $)$ and the hot core position $\left(\left(-6^{\prime \prime},-6^{\prime \prime}\right)\right.$, lower panel $)$

IRAS HIRES and MSX images of the region, a luminosity between $5-15 \times 10^{4} \mathrm{~L}_{\odot}$ is estimated for the hot molecular core.

\section{The cold molecular clump}

Spectra of the cold molecular clump to the north-east of the hot core are shown in Fig. 3. Extremely bright $\mathrm{N}_{2} \mathrm{H}^{+}$ was detected and even emission from $\mathrm{H}_{2} \mathrm{CO}$, probing an embedded "hot corino" within the cold clump. This finding prompted us to observe the fundamental $\mathrm{N}_{2} \mathrm{H}^{+}$rotational transition with the ATCA at high angular resolution. The $\mathrm{N}_{2} \mathrm{H}^{+}(1-0)$ integrated intensity distribution is shown in Fig. 4. $\mathrm{N}_{2} \mathrm{H}^{+}$probes a mid-infrared dark clump with a size of about 1 parsec. With the high angular resolution of ATCA, fragments within this clump with core size of 0.1 to 0.2 parsec can be detected. The $\mathrm{N}_{2} \mathrm{H}^{+}$emission avoids the hot core itself. The ${ }^{14} \mathrm{~N}$ hyperfine structure of the $(1-0)$ lines is partly resolved in the observations and therefore the

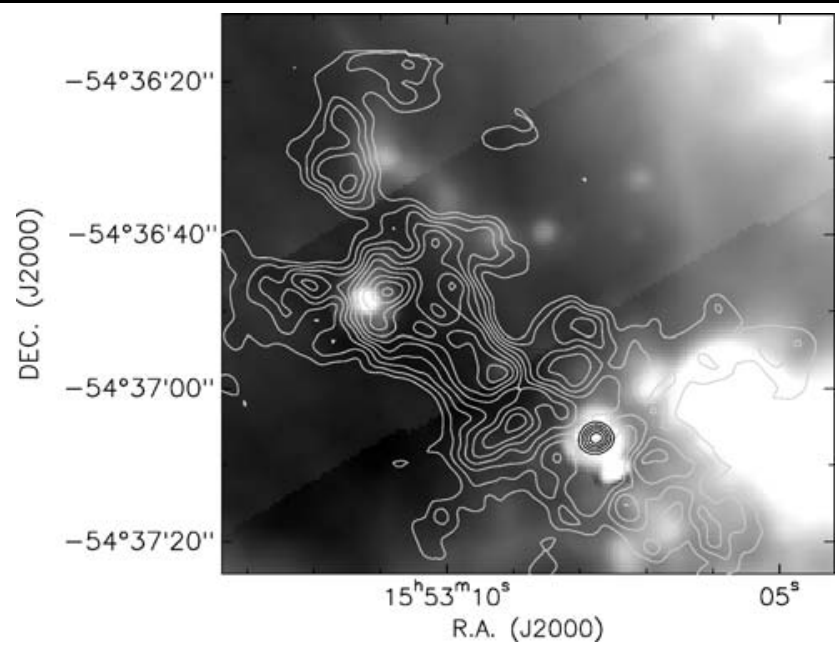

Fig. 4 ATCA $\mathrm{N}_{2} \mathrm{H}^{+}(1-0)$ (grey contours) and $3 \mathrm{~mm}$ continuum (black) with GLIMPSE 8 micron as greyscale background

optical depth and the excitation temperature and, in turn, the $\mathrm{N}_{2} \mathrm{H}^{+}$column density of the cores can be determined. These parameters can be used together with Non-LTE molecular radiative transfer modelling (using $\mathrm{RADEX}_{\text {on-line) }}{ }^{1}$ of the higher excited lines observed with APEX to constrain the density of the cores. To reproduce the strong emission of the higher- $J \mathrm{~N}_{2} \mathrm{H}^{+}$lines, densities of at least $5 \times 10^{6} \mathrm{~cm}^{-3}$ are needed. Together with the observed sizes this points to masses of order $500 \mathrm{M}_{\odot}$, hence similar to the hot molecular core. The observed line widths of $\mathrm{N}_{2} \mathrm{H}^{+}$can be used to estimate the virial masses of the cold cores, which are only several 10 solar masses, hence these cores are likely gravitationally unstable and therefore represent a promising and rare example for massive pre-protocluster cores.

\section{Summary}

The combined APEX/ATCA study of the environs of the hot molecular core G327.3-0.6 shows the power of combining the higher angular resolution of millimeter interferometers with observations of high excitation lines accessible to submillimeter telescopes. The observations resolve the region into several different stages of massive star formation:

- A cold clump harboring promising pre-protocluster cores.

- A luminous hot molecular core offset from a UC HII region, visible in Fig. 4 west of the core.

- A large HII region with bright photon dominated region to the north.

G327.3-0.6 will therefore be an ideal laboratory for ALMA to study and test evolutionary schemes of massive star formation.

\footnotetext{
${ }^{1}$ http://www.sron.rug.nl/ vdtak/radex/radex.php.
} 


\section{References}

Bergman, P.: PhD thesis, Göteborg (1992)

Epchtein, N., Lepine, J.R.D.: Astron. Astrophys. 99, 210 (1981)

Gibb, E., Nummelin, A., Irvine, W.M., Whittet, D.C.B., Bergman, P.: Astrophys. J. 545, 309 (2000)

Goss, W.M., Shaver, P.A.: Austr. J. Phys. Astrophys. Suppl. 14, 1 (1970)

Güsten, R., Nyman, L.Å., Schilke, P., Menten, K., Cesarsky, C., Booth, R.: Astron. Astrophys. 454, L13 (2006)
Nummelin, A., Dickens, J.E., Bergman, P., Hjalmarson, A., Irvine, W.M., Ikeda, M., Ohishi, M.: Astron. Astrophys. 337, 27 (1998)

Schilke, P., Comito, C., Thorwirth, S., Wyrowski, F., Menten, K.M., Güsten, R., Bergman, P., Nyman, L.-Å: Astron. Astrophys. 454, L41 (2006)

Walmsley, M.: In: Revista Mexicana de Astronomia y Astrofisica Conference Series 1, p. 137 (1995)

Wyrowski, F., Menten, K.M., Schilke, P., Thorwirth, S., Güsten, R., Bergman, P.: Astron. Astrophys. 454, L91-L94 (2006) 